\section{Current frontiers in the clinical research of coronary physiology}

\begin{abstract}
Assessment of coronary physiology has potential diagnostic value when combined with invasive angiography for the assessment and treatment of patients presenting with chest pain. In this article, we review the rationale and evidence base $f r$ the measurement of fractional flow reserve, coronary flow reserve and the index f microvascular resistance in stable coronary artery disease and in acute c $r$ nary syndromes. The current and future role of these physiological tests in utine clinical practice is also considered.
\end{abstract}

Keywords: Coronary artery disease, coronary flow reserve, fractional flow reserve, index of microvascular resistance, microvascular function

Coronary artery disease (CAD) is the major cause of premature morbidity and mortality, and in the UK 726,000 men and 393,000 women aged between 35 and 75 suffer from angina pectoris $[1,2]$. Angina is due to a mismatch in the supply or demand of blood flow to the myocardium resulting in the development of ischemia. Factors which may limit myocardial perfusion include hypotension, obstructive CAD and increased microvascular resistance. By angiographic criteria, flowlimiting coronary artery disease is typically associated with a reduction in the diameter of the left main coronary artery by at least $50 \%$ or of any other epicardial artery by at least 70\% [3]. In practice, the relationships between coronary flow and stenosis severity are much more complex and an anatomical assessment of CAD alone is inadequate to accurately identify functionally significant disease.

Angina and inducible ischemia may occur in the absence of a flow-limiting coronary artery stenosis. When the etiology is attributed to coronary microvascular dysfunction then the diagnosis is microvascular angina. However, epicardial CAD and microvascular dysfunction may coexist in the same patient, explaining why angina may persist after successful stenting with percutaneous coronary intervention $(\mathrm{PCl})$.

Recent advances in the assessment of myocardial blood flow based on coronary guidewire pressure and temperature sensors have enabled direct measurement of lesion-level ischemia and related parameters of microvascular function. Compared with $\mathrm{PCl}$ guided by visual interpretation of the angiogram, pressure wire guided $\mathrm{PCl}$ improves clinical outcomes and is cost-effective. This technology also has the potential to provide clinically relevant information in patients with angina and a normal or near normal coronary angiogram.
David Corcoran ${ }^{1,2}$, Colin Berry*,1,2 \& Keith Oldroyd ${ }^{1,2}$

'BHF Glasgow Cardiovascular Research Centre, Institute of Cardiovascular \& Medical Sciences, 126 University Place, University of Glasgow, Glasgow, G12 8TA, Scotland, UK

${ }^{2}$ West of Scotland Heart \& Lung Centre, Golden Jubilee National Hospital, Agamemnon Street, Clydebank, G81 4DY, UK

*Author for correspondence: Colin Berry

West of Scotland Heart \& Lung Centre, Golden Jubilee National Hospital Agamemnon Street, Clydebank, G81 4DY, UK Tel: +440 141330 1671, +4401419515000 Fax: +4401413306794 E-mail: colin.berry@glasgow.ac.uk 
Review Article

\section{Theoretical basis of coronary physiology}

\section{Fractional flow reserve}

Within the physiological range of perfusion pressures, vasoconstriction and vasodilatation of the distal coronary resistance vessels (those with a diameter $<500 \mu \mathrm{m}$ ) preserve myocardial perfusion through auto-regulation of microvascular tone. In the presence of an obstructive epicardial coronary artery stenosis, there is reduced perfusion pressure over the stenosis resulting in compensatory vasodilatation of these autoregulatory resistance vessels [4]. When coronary resistance is minimized with the use of a potent vasodilator, such as adenosine, there is an approximately linear relationship between coronary perfusion pressure and blood flow within the physiological blood pressure range. Therefore, a decrease in pressure across a stenosis leads to a reduction in myocardial blood flow. The ratio of the pressure distal to a coronary artery stenosis $(\mathrm{Pd})$ and aortic pressure $(\mathrm{Pa})$ can then be used to estimate the functional severity of the lesion during hyperemia.

The fractional flow reserve (FFR) is an index of the physiological significance of a coronary stenosis and is defined as the ratio of maximal blood flow in a stenotic artery to normal maximal flow. FFR is calculated as: $F F R=P d / P a$, during maximal hyperemia. When compared with the use of multiple noninvasive tests, the FFR threshold for discriminating lesionlevel ischemia is approximately 0.75 , with a normal value for all arteries of 1.0. In order to increase measurement sensitivity, a threshold of 0.80 has been adopted [5], therefore FFR values between 0.75 and 0.80 are within an intermediate 'gray-zone', in which physician decision-making informed by the totality of clinical information becomes even more important. FFR may be corrected for collateral blood flow by measuring a coronary wedge pressure (FFRmyocardial = FFRcoronary + FFRcollateral) [6]. In all cases, the treatment decision based on FFR should be informed by coronary stenosis characteristics, including lesion location and feasibility of revascularization, and patient factors, such as the presence or absence of symptoms, disease severity and co-morbidities.

\section{Hyperemia}

FFR, by definition, requires a reduction in microvascular tone through inducible coronary hyperemia. Hyperemia may be induced by systemic or intracoronary administration of vasodilator drugs, including adenosine, nicorandil, papverine, sodium nitroprusside or even radiographic contrast media. Currently, adenosine is used most frequently, although nicorandil and papaverine are alternative options that are commonly used in by cardiologists in Asia. Adenosine activates A2 receptor subtypes in vascular smooth muscle resulting in vasodilation and a hemodynamic response to systemic adenosine would be expected [7]. If a submaximal response to adenosine occurs then the FFR may be overestimated resulting in underestimation of the functional significance of a coronary lesion [8]. Theoretically, a submaximal response may also occur in patients with diffuse coronary disease, acute myocardial infarction (MI), myocardial bridging and in patients with marked elevation of central venous pressure (e.g., acute heart failure). Regadenoson is a specific A2A adenosine receptor agonist, unlike adenosine which acts on $A 1, A 2 A, A 2 B$ and $A 3$ receptors. Compared with adenosine, regadenoson is better tolerated but much more expensive, at least out with the USA [9].

\section{Adenosine-free coronary artery lesion assessment: Resting Pd/Pa\& iFR}

Resting translesional coronary pressure gradients have also been proposed as a measure of stenosis severity in order to minimize the use of adenosine and its associated side effects. This concept was first considered many years ago and considered to be diagnostically inferior to hyperemic indices [10]. Nevertheless, resting pressure indices have been revisited and now include resting $\mathrm{Pd} / \mathrm{Pa}$ (whole cardiac cycle) and diastolic indices of coronary pressure, including the instantaneous wave-free ratio (iFR) and diastolic $\mathrm{Pd} / \mathrm{Pa}$.

Although initially proposed as an 'adenosine-free' index with an ischemic threshold of 0.83 , the implementation of iFR has evolved into a hybrid with adenosine and with a different ischemic threshold (0.89) allowing for imprecision in the measurement (range 0.86-0.93). Whereas FFR averages the relative distal coronary pressure over the whole cardiac cycle, iFR measures coronary pressure segmented to a specific part of diastole $(25 \%$ into diastole as defined by the dicrotic notch, to $5 \mathrm{~ms}$ before the end of diastole). Prospective studies of iFR and $\mathrm{Pd} / \mathrm{Pa}$, including VERIFY [11], and a pooled analysis (RESOLVE) [12], have found $\mathrm{iFR}$ and $\mathrm{Pd} / \mathrm{Pa}$ to have similar and moderate diagnostic accuracy compared with FFR.

\section{Fractional flow reserve in stable coronary artery disease}

The 'ischemia hypothesis' supposes that relief of ischemia by coronary revascularization leads to improved clinical outcomes. In patients with stable CAD, the neutral results of the Optimal Medical Therapy with or without PCI for Stable Coronary Disease (COURAGE) and Bypass Angioplasty Revascularization Investigation 2 Diabetes (BARI-2D) clinical trials have called into question the clinical benefits of revascularization by PCI [13-15]. 
In COURAGE, 2287 patients with stable angina and visually assessed severe coronary stenosis were randomized to PCI or no PCI. At a median follow-up of 4.6 years, there was no difference in the composite primary end point of death and MI, or in the secondary end points of death, MI, stroke or hospitalization for acute coronary syndrome. BARI-2D, randomized 2368 patients with both Type 2 diabetes mellitus and stable CAD in a $2 \times 2$ factorial design to either prompt revascularization with intensive medical therapy or intensive medical therapy alone and to undergo either insulin-sensitization or insulin-provision therapy. At 5 years, there was no difference in the primary end point of death from any cause, or the principal secondary end point (a composite of death, MI or stroke), for patients who underwent revascularization compared with intensive medical therapy. However, these studies used angiography alone to assess the functional significance of coronary lesions. This almost certainly led to the incorrect categorization of lesions, resulting in inappropriate deferral of PCI. In addition, PCI of nonischemic lesions exposes the patient to the inherent risks of PCI without any likelihood of clinical benefit and with additional unnecessary cost.

The European Society of Cardiology guidelines give FFR a class 1A recommendation for the functional assessment of coronary artery stenoses of intermediate angiographic severity (50-90\%) in order to identify those lesions that are likely to require revascularization [16], and, partly as a result of this, adoption of FFR has been increasing in the UK [17] and internationally [18].

The Fractional Flow Reserve to Determine the Appropriateness of Angioplasty in Moderate Coronary Stenosis (DEFER) trial was the first prospective randomized trial to demonstrate that patients with negative FFR values (defined as $>0.75$ ) did not benefit from PCI when compared with medical therapy [19]. In DEFER, 325 patients who were planned to undergo PCI underwent FFR measurement. Patients with an FFR $\geq 0.75$ were randomized to deferral of PCI or to undergo PCI, whereas those with an FFR less than 0.75 underwent PCI as planned. There was no significant difference in the primary end point of absence of adverse cardiac events at 24 months follow-up. However, angina improved in patients with an FFR below the ischemic threshold who were treated by PCI.

The Fractional Flow Reserve versus Angiography for Guiding Percutaneous Coronary intervention (FAME) trial tested the hypothesis that PCI guided by FFR measurement in stable patients would alter lesion classification and improve health and economic outcomes [5]. After the decision to undertake PCI based on angiography, 1005 patients with a greater than $50 \%$ stenosis were randomized to FFR-guided PCI (PCI performed if the FFR was $\leq 0.8$ ) or to continue with PCI, as guided by angiography alone. The composite primary outcome of death, MI or repeat revascularization at 1 year was lower in the FFR-guided group than in the angiography- guided group, and this difference was sustained during longer term follow-up at 2 years. The FFRguided strategy also reduced hospital costs. In FAME, approximately $80 \%$ of PCI-treated patients were free of angina after 1-year followup. However, in COURAGE, only $58 \%$ of patients treated with optimal medical therapy (OMT) alone were free of angina at 1 year.

In the Fractional Flow Reserve-Guided PCI versus Medical Therapy in Stable Coronary Disease (FAME-2) clinical trial, FFR was used to discriminate ischemiainducing lesions ( FFR $\leq 0.80$ ) from nonflow-limiting lesions [20]. Twelve-hundred and twenty patients with one or more angiographically severe stenoses were enrolled. In the subset of patients with lesions with an FFR $\leq 0.80$ that were amenable to PCI ( $\mathrm{n}=888$ patients), the randomized strategy involved PCI of all lesions with OMT versus OMT only without PCI. Patients with CAD associated with an FFR greater than 0.80 were not randomized and instead entered a followup registry involving OMT. On the recommendation of the Data and Safety Monitoring Committee, the trial was stopped early because of a between-group difference in the primary outcome (composite of death from any cause, MI and urgent repeat revascularization at 2 years) and that any change to this difference was improbable should further patients be randomized. The difference in the primary outcome event rates was predominantly influenced by an increase in urgent revascularization in patients randomized to OMT alone. After follow-up to 2 years, the observed betweengroup difference in the primary outcome was maintained [21]. Accordingly, the results of the FAME-2 study indicate that patients with symptoms and ischemia derive prognostic benefits from PCI versus medical therapy alone. The results of FAME-2 refute the findings of the COURAGE trial.

In a sub-study of COURAGE, those patients with evidence of inducible ischemia on SPECT imaging $(\geq 10 \%$ ischemic myocardium) who were randomized to PCI had significant reductions in inducible ischemia and in the annual rate of death and MI [22]. This result led to the hypothesis that PCI may improve outcome in those with stable CAD and a moderate-tolarge ischemic burden, and this hypothesis is being tested in the International Study of Comparative Health Effectiveness with Medical and Invasive Approaches (ISCHEMIA, NCT01471522) trial.

The ISCHEMIA trial tests the hypothesis that an initial invasive strategy of coronary angiography followed by PCI if feasible, in addition to OMT, will reduce the primary composite end point of cardiovascular death or nonfatal MI in patients with at least moderate ischemia on stress imaging, compared with an initial conservative strategy of OMT alone with coronary angiography reserved for failure of OMT. The invasive strategy involves FFRguided revascularization of 'intermediate' coronary stenoses (50$80 \%$ severity), routine revascularization for lesions greater than $80 \%$ severity and no revascularization for lesions less than $50 \%$ severity. The trial is currently enrolling patients worldwide. 
In the Synergy between percutaneous coronary intervention with Taxus and cardiac surgery (SYNTAX) study, 1800 patients with left main stem and multivessel CAD were randomized to PCI or Coronary Artery Bypass Grafting (CABG) [23]. SYNTAX demonstrated significantly increased rates of major adverse cardiac or cerebrovascular events at 12 months in those who underwent PCI (17.8 vs $12.4 \%$ for CABG), failing to meet the criterion of noninferiority for PCI. However, coronary lesions were assessed angiographically without FFR measurement. The planned Comparison of Fractional Flow Reserve-Guided Percutaneous Coronary Intervention and Coronary Artery Bypass Graft Surgery in Patients With Multivessel Coronary Artery Disease (FAME 3, NCT02100722) study will randomize 1500 patients with multivessel CAD to FFR-guided PCI or CABG in patients with the primary end point of 1 -year major adverse cardiac event (MACE) rate.

\section{Fractional flow reserve in non-ST-elevation acute coronary syndrome}

Patients with high-risk non-ST-elevation acute coronary syndrome (NSTE-ACS) derive prognostic benefit from early coronary angiography and revascularization [24,25]. Treatment decisions are based on visual interpretation of the coronary angiogram, supported by all other clinical information. Noninvasive stress testing is not recommended in clinical guidelines and so is usually not available. Accordingly, ad hoc treatment decisions may have greater subjectivity in NSTE-ACS patients compared with treatment decisions made by clinicians during the elective management of patients with stable angina.

While overall, the clinical evidence base favors revascularization of obstructive culprit lesions in symptomatic patients, there is a lack of evidence to inform treatment decisions for patients with mild nonflow-limiting culprit lesions. Current practice is based on the premise that PCI in acute coronary syndrome for coronary plaque rupture may reduce the risk of recurrent events. However, in FFR-negative lesions, OMT may be sufficient. Additionally, in NSTE-ACS patients with multi-vessel disease (MVD), it is uncertain whether nonculprit coronary lesions should also undergo revascularization.

A post-hoc analysis of health outcomes in participants in the Acute Catheterization and Urgent Intervention Triage Strategy (ACUITY) clinical trial indicated that incomplete coronary revascularization was associated with a worse prognosis at 1 year [26]. ACUITY was a prospective clinical trial of 13,819 patients with moderate- and high-risk NSTE-ACS who underwent an early invasive management strategy and were randomized to different anticoagulation strategies. Quantitative coronary angiography was performed on 2954 patients who underwent PCI, and depending on the threshold of residual diameter stenosis post-PCI used, incomplete revascularization was present in 17$75 \%$ of patients with NSTE-ACS after PCI. Regardless of the diameter stenosis threshold used, incomplete revascularization was strongly associated with increased MI at 1-year and ischemiadriven unplanned revascularization, with a trend toward increased mortality. The risk of adverse outcome was related to the number of nonrevascularized lesions.

The Fractional Flow Reserve Versus Angiographically Guided Management to Optimize Outcomes in Unstable Coronary Syndromes (FAMOUS-NSTEMI, NCT01764334) clinical trial was designed to address these uncertainties [27,28]. The trial took place in six hospitals in the UK between October 2011 and April 2014. Three-hundred and fifty NSTEMI patients with $\geq 1$ coronary stenosis $\geq 30 \%$ severity were randomized following coronary angiography to FFR-guided or angiography- guided intervention. FFR was measured in culprit and nonculprit lesions in all patients, but in line with the randomized strategy, FFR was disclosed in the FFRguided group but not in the angiographyguided group. In the FFR group, an FFR $\leq 0.80$ was an indication for revascularization by PCI or CABG. The primary outcome was the proportion of patients in each group treated with medical therapy alone. One-thousand-twohundred and ninety-seven patients were screened and 853 of these patients gave informed consent, and 350 of these patients were randomized. Overall, FFR was successfully measured in $100 \%$ of the patients. There were 706 lesions and a guidewire dissection occurred in $2(0.03 \%)$ of these lesions. The primary outcome occurred in $21.7 \%$ of patients in the FFR-guided group and $13.2 \%$ of the angiography-guided group post-randomization, and the lower adoption of revascularization was maintained through to 12 months $(p=0.054)$. Overall, the rate of MACE at 12 months was low ( $\ 9 \%)$ and there were between-group differences in the MACE rates between the groups. Health outcomes and quality of life were similar at 12 months.

\section{Fractional flow reserve in STEMI}

FFR has also been proposed to guide PCI of nonculprit lesions in patients presenting with STEMI. Currently, clinical guidelines recommend that STEMI patients with MVD should have culpritonly PCI unless hemodynamically unstable [3,16]. Recently, the Randomized Trial of Preventive Angioplasty in Myocardial Infarction (PRAMI) trial has challenged this recommendation. In PRAMI, 465 STEMI patients with MVD underwent PCI of culprit lesions, and were then randomized to have no further PCI or to also have preventative PCI of nonculprit lesions greater than 50\% [29]. PRAMI was stopped early due to a statistically significant decreased incidence of the primary end point of death, MI or refractory angina in the preventative PCI group, driven primarily by reduced rate of reinfarction and recurrent ischemia. The Complete versus Lesiononly Primary PCI Trial (CULPRIT) trial addressed a similar question in 296 STEMI patients with multivessel coronary disease. By 12 months, 31 (21.2\%) patients in the infarct artery only group experienced a MACE event compared with $15(10.0 \%)$ in the complete revascularization group [30]. The ongoing Complete versus Culprit-only Revascularization to Treat Multi-vessel Disease After Primary PCI for STEMI (COMPLETE, 
NCT01740479) trial will randomize 3900 patients within 72 hours of the index primary or rescue PCI to culprit only PCI, or PCI of the culprit lesion and additional PCI of nonculprit lesions if FFR $\leq 0.8$. The primary outcome is a composite of cardiovascular death or MI. Given that two independent randomized trials have provided consistent results in favor of multivessel PCI at the time of primary PCI, we think the clinical guidelines committees should reconsider the current guideline recommendation against additional PCI of nonculprit lesions.

\section{Fractional flow reserve in other circumstances}

FFR may be performed following PCI to assess for improvement in flow across the treated lesion. In a multicenter registry of 750 patients, FFR was measured after angiographically successful PCI. Following PCI, an FFR greater than 0.90 was associated with a good long-term result [31].

In diffuse coronary atherosclerotic lesions, an FFR 'pull-back' across serial stenoses helps to discriminate which lesions are associated with a functionally relevant pressure drop, as revealed by a 'step-up' in the FFR as the sensor is withdrawn proximally across the lesion during hyperemia [32].

FFR measurement in angiographically equivocal left main stem stenosis is associated with favorable outcomes. In a cohort study of 213 patients with angiographically equivalent left main stem disease, the prognosis of patients who were medically managed if the FFR greater than 0.80 was similar to those with FFR $\leq 0.80$ who underwent CABG [33].

The SYNTAX score of the complexity of multivessel CAD was modified by the FAME investigators to produce a functional SYNTAX score. Lesions were only included in the score if the FFR $\leq 0.8$. This resulted in a reclassification of angiographically severe but functionally insignificant lesions, with $32 \%$ of patients being moved to lower risk tertiles. The functional SYNTAX score was shown to be a better discriminator than the SYNTAX score alone for MACE at 1-year follow-up [34-36]. However, it should be noted that patients in FAME had less complex CAD compared with those in the SYNTAX trial (mean SYNTAX score 14.8 vs 28.4) and left main stem disease was excluded from the FAME trial (Box 1).

Box 1. Indications for fractional flow reserve
measurement.
Indications for fractional flow reserve
measurement:
- Angiographically intermediate coronary stenosis
- Intermediate left main stem stenosis
- Multivessel disease
- Serial coronary stenoses
- Nonculprit disease in acute coronary syndrome
- Post-percutaneous coronary intervention

Fractional flow reserve in comparison to resting pressure indices

The Adenosine Vasodilatation Independent Stenosis Evaluation (ADVISE) proof of concept study demonstrated that coronary resistance was constant and minimized during the wave-free period [37]. The Classification Accuracy of Pressure-Only Ratios Against Indices Using Flow Study (CLARIFY) compared iFR and FFR to another physiological measure of the severity of stenosis, the hyperemic stenosis resistance (HSR) [38]. HSR is defined as the ratio between the pressure drop across a stenosis and distal peak flow velocity at maximal hyperemia. The use of HSR in clinical practice is limited as intracoronary Doppler flow velocity measurements are not straightforward to acquire in every day practice and their reproducibility can be problematic. CLARIFY showed that iFR and FFR had equivalent agreement with classification of coronary stenosis severity when compared with HSR.

Verification of Instantaneous Wave-Free Ratio and Fractional Flow Reserve for the Assessment of Coronary Artery Stenosis Severity in Everyday Practice (VERIFY) was a prospective study of 205 patients comparing the diagnostic accuracy of iFR to FFR. VERIFY demonstrated a moderate correlation between FFR and iFR, but only a weak correlation in the diagnostic range of greatest uncertainty for decision-making (FFR $=0.6-0.9)$. Furthermore, evidence was provided that iFR was reduced by adenosine.

The Multicenter Core Laboratory Comparison of the Instantaneous Wave-Free Ratio and Resting Pd/Pa with Fractional Flow Reserve (RESOLVE) study was implemented in order to further assess the relationships between iFR, Pd/Pa and FFR. RESOLVE was a retrospective core laboratory-based analysis of coronary pressure recordings, and specifically iFR, whole cycle $\mathrm{Pd} / \mathrm{Pa}$ and FFR in 1768 patients from 15 hospitals [12]. The results of this analysis disclosed a moderate linear correlation between iFR and $\mathrm{Pd} / \mathrm{Pa}$ with FFR as the reference test with a diagnostic accuracy of $80 \%$ for both resting indices.

Subsequently, a hybrid iFR/FFR approach has been proposed. The Adenosine Vasodilator Independent Stenosis Evaluation II (ADVISE II, NCT01740895) was an observational, nonrandomized registry study of 690 patients [39]. The results of this study have been publically presented but have not yet been published. Using a hybrid approach with initial resting pressure measurement, ADVISE II suggested that stenoses with an $\mathrm{iFR} \geq 0.94$ may be deferred while those $\leq 0.85$ can be treated by revascularization. Stenoses with an iFR between 0.86 and 0.93 should have further assessment with FFR to guide decisionmaking. ADVISE II found that iFR characterized correctly $91.6 \%$ of the stenoses in terms of hemodynamic severity outside of the prespecified $\leq 0.85$ and $\geq 0.94$ values, and that a hybrid iFR/FFR approach would avoid adenosine usage in $69.5 \%$ of stenosis.

The ongoing Functional Lesion Assessment of Intermediate Stenosis to Guide Revascularization (DEFINE-FLAIR, NCT02053038) study is comparing FFR-guided versus iFR-guided PCI in 2500 patients with intermediate coronary lesions and the primary 
outcome is major adverse cardiac events (death, MI, unplanned revascularization). DEFINE-FLAIR has a noninferiority design. In the Evaluation of iFR versus FFR in Stable Angina or Acute Coronary Syndrome (iFR SWEDEHEART, NCT02166736) patients with either stable angina or NSTE-ACS and who have an indication for a functional coronary lesion assessment at the time of invasive angiography are randomized to either iFRguided management or FFR-guided management. The primary outcome measure is death, nonfatal MI and unplanned revascularization at 12 months, and this study has a superiority design.

\section{Invasive assessments of microvascular function in patients with} angina

Coronary microvascular dysfunction may provoke typical symptoms of angina and has evidence of inducible myocardial ischemia, without obstructive epicardial CAD. It is proposed that in microvascular angina, the coronary microvasculature is unable to increase coronary blood flow at times of increased myocardial demand. Chest pain occurring in the context of nonobstructive CAD may be designated as 'Syndrome $\mathrm{X}$ ' or potentially misdiagnosed as noncardiac chest pain (Table 1) [40,41].

\begin{tabular}{|l|l|}
\hline \multicolumn{2}{|l|}{ Coronary microvascular disease } \\
\hline Type $\mathbf{1}$ & $\begin{array}{l}\text { Primary, no myocardial or obstructive coronary artery } \\
\text { disease }\end{array}$ \\
\hline Type $\mathbf{2}$ & $\begin{array}{l}\text { In the presence of cardiomyopathy (LVH, HCM, } \\
\text { DCM, amyloidosis) }\end{array}$ \\
\hline Type 3 & In the presence of obstructive CAD \\
\hline Type $\mathbf{4}$ & Postcoronary intervention \\
\hline Type 5 & Postcardiac transplantation \\
\hline $\begin{array}{l}\text { DCM: } \\
\text { cardiomyopathy; LVH: Left ventricular hypertrophy. }\end{array}$ \\
\hline
\end{tabular}

Table 1. Clinical classification of coronary microvascular dysfunction.

Angina may occur even in patients with nonobstructive CAD. For example, in the FAME-2 trial, $13.6 \%$ of symptomatic patients initially enrolled had an FFR greater than 0.8 . These patients may have been misdiagnosed with angina, but in those patients with objective evidence of ischemia, microvascular dysfunction is a possible explanation. Microvascular disease is more common in women, and is associated with an adverse prognosis $[42,43]$.

Coronary blood flow and myocardial perfusion are regulated by arterioles $(<200 \mu \mathrm{m})$ within the myocardium and prearterioles $(<500 \mu \mathrm{m})$ within the epicardium, with these vessels contributing 50 and $25 \%$ respectively to total coronary vascular resistance. In the absence of significant stenosis, the epicardial arteries act as conductance vessels and offer little resistance to flow. Arterioles and precapillaries have vascular smooth muscle cells within their walls, which can influence vascular tone, and this can be affected by flowrelated vasoreactivity, intraluminal pressure changes and metabolic activity. The pathophysiology of coronary microvascular disease involves a reduction in the number of microvascular arterioles, and potentially microvascular hypertrophy which may reduce the radius and lumen area of these blood vessels [44-46]. Accordingly, microvascular resistance increases according to Poiseuille's Law. The coronary microvasculature may be assessed by invasive and noninvasive methods.

\section{Coronary flow reserve}

Traditionally, microvascular disease has been difficult to diagnose as diagnostic tests have been limited. Microvascular capillary numbers correlate inversely with symptoms and vasodilator capacity as measured by stress testing or coronary flow reserve (CFR). CFR represents the vasodilator capacity of the coronary microcirculation. It can be measured by indicator thermodilution using the coronary pressure wire, where the pressure transducer is the distal thermistor and the pressure wire shaft is the proximal thermistor. CFR is then calculated as the ratio of resting (Trest) to hyperaemic (Thyp) transit time as with a $3 \mathrm{ml}$ bolus of room temperature saline, where CFRthermo $=\mathrm{T}$ rest $/$ Thyp. CFR values are normally above 4 , with values less than 2 indicative of significant microvascular dysfunction in the absence of epicardial coronary stenosis. CFR is not measured in routine practice because of problems with reproducibility and uncertainties around its clinical and prognostic significance. Recently however, discordance between FFR and CFR measurements in those patients with an FFR greater than 0.8 but an abnormal CFR value, has been associated with microvascular disease and poor prognosis [47].

\section{Pressure wire measurement of microvascular function}

The pressure wire can also be used to measure indices of microvascular function, which unlike CFR is independent of coronary stenosis severity, and has acceptable reproducibility. The index of microvascular resistance (IMR) is a measurement of microcirculatory function. IMR is calculated from distal coronary pressure $(\mathrm{Pd})$ multiplied by the mean transit time of a $3 \mathrm{ml}$ bolus of room temperature saline during coronary hyperaemia (Thyp) induced by intravenous adenosine (IMR = Pd x Thyp), and as hyperemic transit time is inversely correlated with flow it provides a quantitative measure of coronary microvascular resistance [48]. An IMR less than 25 is normal, with values greater than this consistent with microvascular dysfunction [49]. IMR is not significantly affected by epicardial coronary artery stenosis and therefore allows for the specific measurement of microvascular resistance [50]. However, in a severe epicardial coronary artery stenosis, there may be an associated recruited collateral supply, and in this case the coronary wedge pressure and venous pressure must be accounted for (IMRcorr = PaHyp $\mathrm{x}$ TmnHyp $\mathrm{x}$ [(PdHyp - Pw) / (PaHyp - Pw)]) or resistance will be overestimated [51]. As IMR is measured at maximal hyperemia, it is independent of hemodynamic variations, and therefore has less variability and better repeatability than CFR (Figure 1) [52]. 


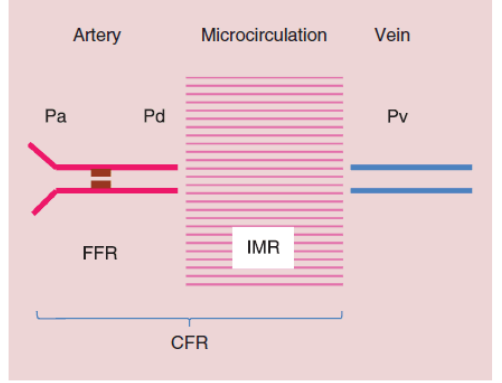

Figure 1. Relationship between coronary flow reserve, fractional flow reserve and index of microvascular resistance.CFR: Coronary flow reserve; FFR: Fractional flow reserve; IMR: Index of microvascular resistance; Pa: Aortic pressure; Pd: Pressure distal to coronary stenosis; Pv: Central venous pressure.

Another novel parameter of microvascular function is the resistive reserve ratio (RRR). This is defined as the baseline microvascular resistance (Pd x Trest) indexed to microvascular resistance during hyperemia (IMR), with vasodilation resulting in a fall in resistance. This is a measure of the vasodilatory capacity of the coronary microcirculation in response to a pharmacological hyperemic stimulus, with an RRR greater than 1 being normal. The RRR has a negative correlation with troponin in patients presenting with STEMI, which may be explained by the severity of the microvascular dysfunction (Figure 2) [53].

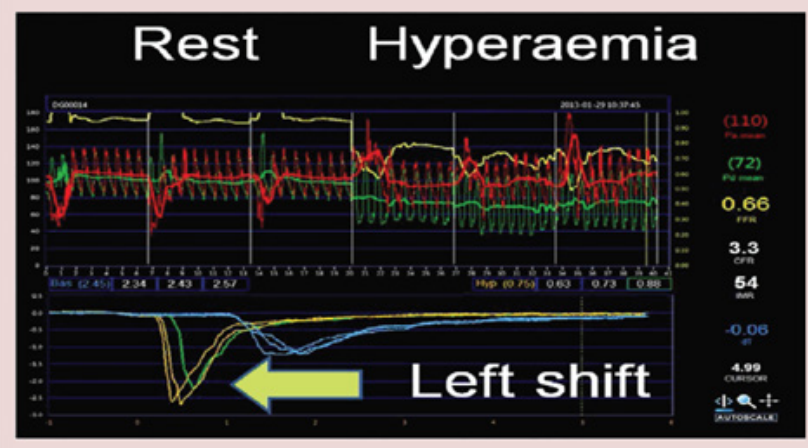

Figure 2. Example of fractional flow reserve, index of microyascular resistance and coronary flow reserve measurement using a pressure wire.

It is not known whether therapeutic reduction of IMR or treatment decisions based on IMR or RRR may confer symptomatic or prognostic benefit. Therapeutic interventions to reduce microvascular injury at the time of myocardial intervention and PCI are being investigated, including vasodilatory therapy (calcium channel blockers, sodium nitroprusside and adenosine), antiplatelet agents (glycoprotein IIb/ IIIa) and mechanical agents (thrombectomy and distal embolic protection devices). The intracoronary abciximab and aspiration thrombectomy in patients with large anterior myocardial infarction (INFUSEAMI) trial showed a modest effect on infarct size when thrombectomy and intracoronary abciximab were used in combination, but not as monotherapies [54]. These results support the rationale for new approaches to identify patients with acute coronary microvascular injury, for example by IMR measurement, which might be reversible and amenable to treatment.
IMR can be measured in patients with chronic stable angina [55], and also in those with acute MI in which it adds prognostic significance [56]. Patients with microvascular obstruction postMI as measured on cardiac magnetic resonance have been shown to have higher IMR values after PCI than in those who did not have evidence of microvascular obstruction [57]. Microvascular resistance after primary PCI for STEMI is an independent predictor of myocardial salvage (Box 2) [58].

Box 2 . Indications for the measurement
of coronary flow reserve and index of
microvascular resistance.
Coronary flow reserve
- Assessment of coronary vascular function.
- Diagnosis of microvascular angina.
Index of microvascular resistance
- Assessment of microvascular function.
- Prognostic assessment in acute coronary syndrome.

Other methods for assessing coronary microvascular function

The coronary Doppler guidewire is able to quantify coronary blood flow and thus derive CFR from the ratio of hyperemic and baseline average peak velocity. However, its use is limited by a high intrinsic variability and it is not readily available [59]. Noninvasive assessment imaging modalities have also been used to assess the coronary microvasculature.

Positron emission tomography (PET) perfusion imaging is the gold standard for noninvasive quantification of myocardial blood flow. PET uses the administration of radiolabeled tracers and dynamic measurement of tracer concentration in the myocardium at stress and rest allows for the quantification of CFR [60]. However, PET imaging is expensive and not widely available for clinical practice. Coronary vascular resistance (CVR) can be estimated by dividing arterial blood pressure by the PET-derived myocardial blood flow at hyperemia. However, in the presence of an epicardial coronary artery stenosis, CVR does not differentiate between increased CVR due to epicardial stenosis or microvascular disease [61].

Contrast-enhanced cardiac magnetic resonance (CMR) can accurately demonstrate infarct size with late gadolinium enhancement imaging. If present, a hypoenhanced core represents microvascular obstruction which can then be quantified. Oxygenationsensitive CMR is an experimental technique to assess myocardial blood flow, which exploits the intrinsic contrast of deoxygenated hemoglobin in blood which can be used to quantify myocardial blood flow [62]. The technique has been validated against FFR in patients with CAD [63].

Additionally, acetylcholine provocation testing has been used to assess the coronary microvasculature and can demonstrate vasomotor abnormalities in conditions such as Prinzmetal's variant angina, but is not easily available in clinical practice (Figure 3) 


\section{Review Article}

[64].

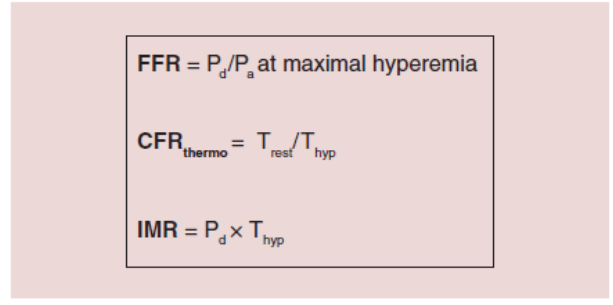

Figure 3. Calculation of coronary flow reserve, fractional flow reserve and index of microvascular resistance.CFR : Coronary flow reserve; FFR: Fractional flow reserve; IMR: Index of microvascular resistance; $P_{\mathrm{a}}$ : Aortic pressure; $\mathrm{P}_{\mathrm{d}}$ : Pressure distal to coronary stenosis; $T_{\text {hyp }}$ : Hyperaemic transit time; $T_{\text {rest }}$ Resting transit time.

\section{Conclusion/Future perspective}

The use of FFR in daily clinical practice is increasing and functional rather than anatomical revascularization is associated with improved clinical outcomes for patients in landmark trials such as DEFER, FAME and FAME-2. In the future, the ISCHEMIA and FAME 3 trials will further define its use in the management of $\mathrm{CAD}$. The diagnosis and management of patients with angina who have no obstructive CAD at angiography remains a challenge. IMR and RRR may allow differentiation of patients with microvascular dysfunction from noncardiac chest pain. In the future, this may allow the targeting of potential therapeutic interventions such as vasodilator therapy and also be used to assess response to therapy.

\section{Financial \& competing interests disclosure}

Professor Berry has acted as a consultant for St. Jude Medical based on a contract with the University of Glasgow. Professor Oldroyd has received consultant and speaker fees from St. Jude Medical and Volcano Corporation which manufacture pressure wires. Dr Corcoran is supported by a BHF Clinical Research Training Fellowship. The authors have no other relevant affiliations or financial involvement with any organization or entity with a financial interest in or financial conflict with the subject matter or materials discussed in the manuscript apart from those disclosed.

No writing assistance was utilized in the production of this manuscript.

\section{Executive summary}

- Theoretical basis of coronary physiology.

- Fractional flow reserve (FFR).

- Hyperemia.

- Adenosine-free coronary artery lesion assessment: resting $\mathrm{Pd} / \mathrm{Pa}$ and iFR.

- FFR in stable coronary artery disease.

- FFR in non ST-elevation acute coronary syndrome.

- FFR in STEMI.

- FFR in comparison to resting pressure indices.
- Invasive assessments of microvascular function in patients with angina.

- Coronary flow reserve.

- Pressure wire measurement of microvascular function.

- Other methods for assessing coronary microvascular function.

- Future perspective.

\section{References}

1. Cooper A, Calvert N, Skinner J et al. Chest pain of recent onset: assessment and diagnosis of recent onset chest pain or discomfort of suspected cardiac origin. National Clinical Guideline Centre for Acute and Chronic Conditions. NICE- 95(2010). London, UK www.nice.org.uk

2. Townsend N, Wickramasinghe K, Bhatnagar P et al. Coronary Heart Disease Statistics. British Heart Foundation. London, UK (2012). www.bhf.org.uk

3. Levine GN, Bates ER, Blankenship JC et al. 2011 ACCF/ AHA/SCAI Guideline for Percutaneous Coronary Intervention. A report of the American College of Cardiology Foundation/American Heart Association Task Force on Practice Guidelines and the Society for Cardiovascular Angiography and Interventions. J. Am. Coll. Cardiol. 58(24), e44-e122 (2011).

4. van de Hoef TP, Meuwissen M, Piek JJ. Fractional flow reserve and beyond Heart 99(22), 1699-1705 (2013).

5. Tonino PA, De Bruyne B, Pijls $\mathrm{NH}$ et al. FAME Study Investigators. Fractional flow reserve versus angiography for guiding percutaneous coronary intervention. N. Engl. J. Med. 360(3), 213-224 (2009).

6. Pijls N. Fractional Flow Reserve to Guide Coronary revascularization. Circ. J 77, 561-569 (2013).

7. van de Hoef TP, Meuwissen M, Escaned J et al. Fractional flow reserve as a surrogate for inducible myocardial ischaemia. Nat. Rev. Cardiol. 10(8), 439452 (2013).

8. Pijls NH1, Tanaka N, Fearon WF. Functional assessment of coronary stenosis: can we live without it? Eur. Heart J. 34(18), 1335-1344 (2013).

9. Prasad A, Zareh M, Doherty R et al. Use of regadenoson for measurement of fractional flow reserve. Catheter Cardiovasc. Interv. 83(3), 369-374 (2014).

10. Gould KL, Lipscomb K, Hamilton GW. Physiologic basis for assessing critical coronary stenosis. Instantaneous flow response and regional distribution during coronary hyperemia as measures of coronary flow reserve. Am. J. Cardiol. 33(1), 87-94 (1974).

11. Berry C, van't Veer M, Witt N et al. VERIFY (VERification of Instantaneous Wave-Free Ratio and Fractional Flow Reserve for the Assessment of Coronary Artery Stenosis Severity in EverydaY Practice): a multicenter study in consecutive patients. J. Am. Coll. Cardiol. 61(13), 1421-1427 (2013).

12. Jeremias A, Maehara A, Généreux $P$ et al. Multicenter Core Laboratory Comparison of the Instantaneous Wave-Free Ratio and Resting Pd/Pa with Fractional Flow Reserve: The RESOLVE Study. J. Am. Coll. Cardiol. 63(13), 1253-1261 (2014).

13. Fox KA. COURAGE or FAME..? Who should have percutaneous coronary intervention in stable coronary artery disease? Heart 99(7), 442-444 (2013).

14. Boden WE, O’Rourke RA, Teo KK et al. COURAGE Trial Research Group. Optimal medical therapy with or without PCI for stable coronary disease. N. Engl. J. Med. 356(15), 1503-1516 (2007). 


\section{Review Article}

15. BARI 2D Study Group, Frye RL, August P et al. A randomized trial of therapies for type 2 diabetes and corona y artery disease. N. Engl. J. Med. 360(24), 2503-2515 (2009) .

16. Montalescot G, Sechtem U, Achenbach S et al. 2013 ESC guidelines on the management of stable co ona y a te y disease. The Task Force on the management of stable coronary disease of the European Society. Eur. Hea t J. 34(38), 2949-3003 (2013).

17. Ludman PF. BCIS 2012 Audit data. www.bcis. rg.uk/pages/ page box contents. asp? pageid $=780 \&$ navcatid $=11$

18. Park SJ, Ahn JM, Park GM et al. Trends in the utc mes of percutaneous coronary in ervention with the routine incorporation of frac ional flow reserve in real practice. Eur. Heart J. 34(43), 3353-3361 (2013).

19. Bech GJ, De Br yne B, Pijls NH et al. Fractional flow reserve to determine the appropriateness of angioplasty in moderate coronary stenosis: a randomized trial. Circulation 103(24), 2928-2934 (2001).

20. De Bruyne B Pijls NH, Kalesan B et al. FAME 2 Trial Investigators. Fractional flow reserve-guided PCI versus medical therapy in stable coronary disease. N. Engl. J. Med. 367(11), 991-1001 (2012).

21. De Bruyne B, Fearon WF, Pijls NH et al. Fractional flow reserve-guided PCI for stable coronary artery disease. N. Engl. J. Med. 371(13), 1208-1217 (2014).

22. Shaw LJ, Berman DS, Maron DJ. COURAGE Investigators. Optimal medical therapy with or without percutaneous coronary intervention to reduce ischemic burden: results from the Clinical Outcomes Utilizing Revascularization and Aggressive Drug Evaluation (COURAGE) trial nuclear substudy. Circulation 117(10), 1283-1291 (2008).

23. Serruys PW, Morice MC, Kappetein AP et al. SYNTAX investigators. Percutaneous coronary intervention versus coronary artery bypass grafting for severe coronary artery disease. N. Engl. J. Med. 360(10), 961-972 (2009).

24. Fox KA, Clayton T, Damman P et al. FIR Collaboration. Long-term outcome of a routine versus selective invasive strategy in patients with non-ST-segment elevation acute coronary syndrome a meta-analysis of individual patient data. J. Am. Coll. Cardiol. 55, 2435-2445 (2010).

25. Mehta SR, Granger CB, Boden WE et al. TIMACS Investigators. Early versus delayed invasive intervention in acute coronary syndromes. N. Engl. J. Med. 360, 2165-2175 (2009).

26. Garcia S, Sandoval Y, Roukoz H et al. Outcomes after complete versus incomplete revascularization of patients with multivessel coronary artery disease: a meta-analysis of 89,883 patients enrolled in randomized clinical trials and observational studies. J. Am. Coll. Cardiol. 62(16), 1421-1431 (2013).

27. Berry C, Layland J, Sood A et al. Fractional flow reserve versus angi graphy in guiding management to optimize outc mes in $\mathrm{n} n-\mathrm{ST}$-elevati n myocardial infarction (FAMOUS-NSTEMI): rati nale and design of a randomized c ntr lled clinical trial. Am. Heart J. 166(4), 662-668 (2013).

28. Layland J, Oldr yd KG, Curzen $\mathrm{N}$ et al. Fractional flow ese ve vs. angi graphy in guiding management to optimize utc mes in $\mathrm{n} n$-ST-segment elevation myocardial infa ction: the British Heart Foundation FAMOUS-NSTEMI randomized trial. Eur. Heart J. 36(2) 100-111 (2015).

29. Wald DS, Morris JK, Wald NJ et al. Randomised trial of preventive angioplasty in myocardial infarction. N. Engl. J. Med. 369(12), 1115-1123 (2013).
30. Gershlick AH. The Complete versus Lesion-only Primary PCI Trial. ESC Congress 2014 Hot Line IV: Myocardial infarction. Presented at: ESC Congress 2014. Barcelona,Spain, 3 September 2014.

31. Pijls NH, Klauss V, Siebert U et al. Fractional Flow Reserve (FFR) Post-Stent Registry Investigators. Coronary pressure measurement after stenting predicts adverse events at follow-up: a multicenter registry. Circulation 105, 2950 2954 (2002).

32. Pijls NH, Kern MJ, Yock PG et al. Practice and potential pitfalls of coronary pressure measurement. Catheter Cardiovasc. Interv. 49, 1-16 (2000).

33. Hamilos M Muller O, Cuisset $\mathrm{T}$ et al. Long-term clinical outcome after fractional flow reserve-guided treatment in patients with angiographically equivocal left main coronary artery stenosis. Circulation 120, 1505-1512 (2009).

34. Kleiman NS. Bringing it all together: integration of physiology with anatomy during cardiac catheterization. J. Am. Coll. Cardiol. 58(12), 1219-1221 (2011).

35. Nam CW, Mangiacapra F, Entjes R et al. FAME Study Investigators. Functional SYNTAX score for risk assessment in multivessel coronary artery disease. J. Am. Coll. Cardiol. 58(12), 1211-1218 (2011).

36. Farooq V, Head SJ, Kappetein AP et al. Widening clinical applications of the SYNTAX Score. Heart 100(4), 276-287 (2014).

37. Sen S, Escaned J, Malik IS et al. Development and validation of a new adenosine-independent index of stenosis severity from coronary wave-intensity analysis: results of the ADVISE (ADenosine Vasodilator Independent Stenosis Evaluation) study. J. Am. Coll. Cardiol. 59(15), 1392-1402 (2012).

38. Sen S1, Asrress KN, Nijjer S et al. Diagnostic classification of the instantaneous wave-free ratio is equivalent to fractional flow reserve and is not improved with adenosine administration. Results of CLARIFY (Classification Accuracy of Pressure-Only Ratios Against Indices Using Flow Study). J. Am. Coll. Cardiol. 61(13), 1409-1420 (2013).

39. Escaned J. Adenosine Vasodilator Independent Stenosis Evaluation II. Presented at TCT 2013. San Francisco, CA, USA. 30 October 2013.

40. Marzilli M, Merz CN, Boden WE et al. Obstructive coronary atherosclerosis and ischemic heart disease: an elusive link! J. Am. Coll. Cardiol. 60(11), 951956 (2012).

41. Herrmann J, Kaski JC, Lerman A. Coronary microvascular dysfunction in the clinical setting: from mystery to reality. Eur. Heart J. 33(22), 2771-2782 (2012).

42. Johnson BD, Shaw LJ, Buchthal SD et al. Prognosis in women with myocardia ischemia in the absence of obstructive coronary disease: results from the National Institutes of Health-National Heart, Lung, and Blood InstituteSponsored Women's Ischemia Syndrome Evaluation (WISE). Circulation 109(24), 2993-2999 (2004).

43. Suwaidi JA, Hamasaki S, Higano ST et al. Long-term follow-up of patients with mild coronary artery disease and endothelial dysfunction. Circulation 101, 948-954 (2000).

44. Campbell DJ, Somaratn JB, Jenkins AJ et al. Differences in myocardial structure and coronary microvasculature between men and women with coronary artery disease. Hypertensi n 57, 186-192 (2011).

45. Mundhenke M, Schwartzkopff B, Strauer BE. Structural analysis of arteriolar and myocardial remodelling in $\mathrm{t}$ e subendocardial region of patients with ypertensive eart disease and hypertrophic cardiomyopa y. Virc ows Arch. 


\section{Review Article}

431(4), 265-273 (1997).

46. Escaned J, Flores A, Garcia-Pavia P et al. Assessment of microcirculatory remodeling wi $\mathrm{h}$ in racoronary flow velocity and pressure measurements: validation with endomyocardial sampling in cardiac allografts. Circ lation 120, 1561-1568 (2009).

47. van de Hoef TP, van Lavieren M, Damman P et al. Physiological Basis and long-term clinical outcome of discordance between fractional flow reserve and coronary flow velocity reserve in coronary stenoses of intermediate severity. Circ. Cardiovasc. Interv. 7(3), 301-311 (2014).

48. Fearon WF, Balsam LB, Farouque HM et al. Novel index for invasively assessing the coronary microcirculation. Circulation 107(25), 3129-3132 (2003).

49. Kobayashi Y, Fearon WF. Invasive coronary microcirculation assessment-current status of index of microcirculatory resistance. Circ. J. 78(5), 10211028 (2014).

50. Ng MK, Yeung AC, Fearon WF. Invasive assessment of the coronary, microcirculation: superior reproducibility and less hemodynamic dependence of index of microcirculatory resistance compared with coronary flow reserve. Circulation 113, 2054-2061 (2006).

51. Fearon WF, Aarnoudse W, Pijls NH et al. Microvascular resistance is not influenced by epicardial coronary artery stenosis severity: experimental validation. Circulation 109, 2269-2272 (2004).

52. Ng MK, Yeung AC, Fearon WF. Invasive assessment of the coronary microcirculation: superior reproducibility and less hemodynamic dependence of index of microcirculatory resistance compared with coronary flow reserve. Circulation 113(17), 2054-2061 (2006).

53. Layland J, Carrick D, McEntegart M et al. The vasodilatory capacity of the coronary microcirculation is preserved in selected patients with NSTEMI. Circ. Cardiovasc. Intervent. 6(3), 231-236 (2013).

54. Stone GW, Maehara A, Witzenbichler B et al. Intracoronary abciximab and aspiration thrombectomy in patients with large anterior myocardial infarction: the INFUSE - AMI randomized trial. JAMA 307(17), 1817-1826 (2012) .

55. Yong AS, Ho M, Shah MG et al. C $\mathrm{r}$ nary microcirculatory resistance is independent $\mathrm{f}$ epicardial sten sis. Circ. Cardiovasc. Interv. 5, 103-108 (2012).

56. Fearon WF, L w AF, Y ng AS et al. Prognostic value of the index of mic $\mathrm{ci}$ culat y esistance measured after primary percutaneous $\mathrm{c}$ na $\mathrm{y}$ interventi $\mathrm{n}$. Circulation 127(24), 2436-2441 (2013).

57. McGeoch R, Watkins S, Berry C et al. The index of microcirculatory resistance measured acutely predicts the extent and severity of myocardial infarction in patients with ST-segment elevation myocardial infarction. JACC Ca diovasc. Interv. 3(7), 715-722 (2010).

58. Payne AR, Berry C, Doolin O et al. Microvascular resistance p edicts myocardial salvage and infarct characteristics in ST-elevation myocardial infarction. J. Am. Heart Assoc. 1(4), e002246 (2012).

59. Pijls NH, De Bruyne B, Smith L et al. Coronary thermodilution to assess flow reserve: validation in humans. Circulation 105(21), 2482-2486 (2002).

60. Gould KL, Johnson NP, Bateman TM et al. Anatomic versus physiologic assessment of coronary artery disease. Role of coronary flow reserve, fractional flow reserve, and positron emission tomography imaging in revascularization decision-making. J. Am. Coll. Cardiol. 62(18), 1639-1653 (2013).

61. Amier RP, Teunissen PF, Marques KM et al. Invasive measurement of coronary microvascular resistance in patients with acute myocardial infarction treated by primary PCI. Heart 100, 13-20 (2014).

62. Friedrich MG, Karamitsos TD. Oxygenation-sensitive cardiovascular magnetic resonance. J. Cardiovasc. Magn. Reson. 15, 43 (2013).

63. Walcher T, Manzke R, Hombach V et al. Myocardial perfusion reserve assessed by T2-prepared steady-state free precession blood oxygen level-dependent magnetic resonance imaging in comparison to fractional flow reserve. Circ. Cardiovasc. Imaging 5(5), 580-586 (2012).

64. Ong P, Athanasiadis A, Sechtem U. Patterns of coronary vasomotor responses to intracoronary acetylcholine provocation. Heart 99(17), 1288-1295 (2013). 